\title{
The population genetics of sporophytic self- incompatibility in Senecio squalidus L. (Asteraceae) I: $S$ allele diversity in a natural population
}

\author{
AC Brennan ${ }^{1}$, SA Harris ${ }^{1}$, DA Tabah ${ }^{2}$ and SJ Hiscock ${ }^{2}$ \\ ${ }^{1}$ Department of Plant Sciences, University of Oxford, South Parks Road, Oxford OX1 3RB, UK; ${ }^{2}$ School of Biological Sciences, \\ University of Bristol, Woodland Road, Bristol BS8 1UG, UK
}

Twenty-six individuals of the sporophytic self-incompatible (SSI) weed, Senecio squalidus were crossed in a full diallel to determine the number and frequency of $S$ alleles in an Oxford population. Incompatibility phenotypes were determined by fruit-set results and the mating patterns observed fitted a SSI model that allowed us to identify six $S$ alleles. Standard population $S$ allele number estimators were modified to deal with $S$ allele data from a species with SSI. These modified estimators predicted a total number of approximately six $S$ alleles for the entire Oxford population of $S$. squalidus. This estimate of $S$ allele number is low compared to other estimates of $S$ allele diversity in species with SSI. Low $S$ allele diversity in $S$. squalidus is expected to have arisen as a consequence of a disturbed population history since its introduction and subsequent colonisation of the British Isles. Other features of the SSI system in S. squalidus were also investigated: (a) the strength of self-incompatibility response; (b) the nature of $S$ allele dominance interactions; and (c) the relative frequencies of $S$ phenotypes. These are discussed in view of the low $S$ allele diversity estimates and the known population history of $S$. squalidus.

Heredity (2002) 89, 430-438. doi:10.1038/sj.hdy.6800159

Keywords: Senecio squalidus; sporophytic self-incompatibility; population genetics; $S$ allele; $S$ allele diversity

\section{Introduction}

Senecio squalidus L. (Oxford Ragwort) is a short-lived perennial weed with a strong system of self-incompatibility (SI) (Abbott and Forbes, 1993). Like other members of the Asteraceae, SI in S. squalidus is sporophytic (Hiscock, 2000a, b). Self-incompatibility serves to prevent self-fertilisation and to restrict inbreeding amongst related plants, where incompatibility interactions are often controlled through the activity of a single $S$ locus (reviewed in Hiscock and Kues, 1999). The $S$ locus is a genetic region of suppressed recombination that appears to consist of many linked genes expressed in either maternal or paternal reproductive tissue with specific male and female gene products interacting to elicit the SI response that prevents fertilization (Hiscock, 2002). In SSI, the pollen apparently expresses both $S$ alleles present in the parental sporophyte, whereas in the other common form of SI, gametophytic self-incompatibility (GSI), pollen grains express only one $S$ allele encoded by the haploid, male (gametophyte) genome. These differences in the apparent expression of $S$ alleles in pollen between GSI and SSI systems have important consequences for the reproductive and evolutionary dynamics of species that possess either SI system. In GSI systems, half-compatible phenotypes result from crosses between plants sharing one $S$ allele,

SJ Hiscock, School of Biological Sciences, University of Bristol, Woodland Road, Bristol BS8 1UG, UK. E-mail: simon.hiscock@bristol.ac.uk Received 17 December 2001; accepted 16 July 2002 whereas in SSI systems, such crosses are usually fully incompatible. Strict codominance between $S$ alleles is necessary for functional GSI, whereas in SSI there are no restrictions on the evolution of complex dominance interactions between $S$ alleles and dominance hierarchies between $S$ alleles form an intrinsic part of most SSI systems (Hiscock and Kues, 1999). In SSI systems, dominance interactions allow for the natural occurrence of individuals homozygous for recessive $S$ alleles, which are absent in normally functioning GSI systems (Stevens and Kay, 1989). In SI systems, mate availability (MA, defined as the average proportion of compatible mates in a population) is maximised and mating system equilibrium reached, at equal $S$ phenotype frequencies (isoplethy) (Finney, 1952). For GSI systems, where dominance interactions between $S$ alleles are absent, isoplethy is equivalent to equal $S$ allele frequencies, whereas in SSI systems, isoplethy results in $S$ allele frequencies that are dependent on the dominance interactions between the $S$ alleles, such that the more recessive alleles occur at higher frequencies than dominant $S$ alleles (Byers and Meagher, 1992; Schierup et al, 1997).

New $S$ alleles, complementary sets of male and female $S$ genes, are believed to evolve very rarely and only under limited selective scenarios, including temporary breakdown of SI, or through dual-specificity alleles (Matton et al, 1999; Uyenoyama et al, 2001). A fundamental consequence of SI is that the selective advantage of an $S$ allele is inversely proportional to its frequency (negative frequency dependent selection) such that rare or new $S$ alleles are favoured and maintained in popu- 
lations for extended periods of evolutionary time (Ioerger et al, 1990; Richman et al, 1996a). Negative frequency dependent selection results in the accumulation of $S$ allele number despite the slow rate of $S$ allele evolution, making the $S$ locus one of the most polymorphic loci known, comparable with the MHC locus of mammals and the mating-type loci of fungi (Hiscock and Kues, 1999). An equilibrium balance will eventually be reached between the slow rate of evolution of new $S$ alleles and their slow rate of loss. It is believed that the interaction between these evolutionary processes with particular SI systems and life history strategies will result in a characteristic species-specific $S$ allele number (Richman et al, $1996 a, b)$. Estimates of $S$ allele number range from 15 to 66 for GSI species and from 33 to 49 S alleles for SSI species (Lawrence, 2000). Trifolium species represent a notable exception to these values, with estimated $S$ allele number as high as 193. Unsurprisingly, species-level $S$ allele estimates, other than for Trifolium, are not much higher than population-level $S$ allele estimates (12 to 45 for GSI species, and 22 to 43 for SSI species, Lawrence, 2000) because inter-population migration and negative frequency dependent selection will effectively maintain numbers of $S$ alleles in a population above that expected from individual population size alone (Wright, 1939).

In the British Isles, S. squalidus is commonly found in disturbed, urban habitats with well-drained soils and along roadsides and on railway lines. The introduction of $S$. squalidus and its subsequent spread in Britain has been well documented (Abbott, 1992; Harris, 2002). S. squalidus was first introduced into Britain, via the Duchess of Beaufort's Gardens at Badminton and the Oxford Botanic Garden, about 300 years ago from Mount Etna, Sicily (Harris, 2002). Following its escape from the Botanic Garden it became established in Britain in the early 19 th century. From the mid-19th century onwards, the developing railway network provided the 'Oxford Ragwort' with many lanes of suitable habitat in the form of clinker beds and it went on to colonise successfully other British cities. Today, S. squalidus is found in most cities and towns throughout Britain, as far north as the Great Glen in Scotland, and also parts of Ireland (Harris, 2002). The recent history of introduction and colonisation by $S$. squalidus in Britain is likely to have caused a loss of genetic diversity and the establishment of appropriate selective pressures for a breakdown of its SI system. However, studies of SI in S. squalidus demonstrate quite clearly that it has a fully functional SSI system (Abbott and Forbes, 1993; Hiscock, 2000a, b).

Here we report the first detailed population genetic study of the mating system of $S$. squalidus. The aims of this study were to determine if there is any variation in the strength of SSI and to estimate $S$ allele diversity in a typical British population of $S$. squalidus.

\section{Materials and methods}

\section{Plants}

Twenty-six S. squalidus seedlings (Ox1-Ox27, excl. Ox7) were collected from a wild population growing within a $0.5 \mathrm{~km}$ radius of Oxford railway station (grid ref.: SP505064) in March 1999. The seedlings were grown up and maintained in a glasshouse in $20 \mathrm{~cm}$ pots of soilbased potting compost with regulated watering and $16 \mathrm{~h}$ day lighting. When required, individuals were propagated by taking stem cuttings.

\section{Pollinations}

Fruit (achene)-set was chosen as a reliable measure of compatibility phenotype in S. squalidus because incompatible pollinations usually result in little or no fruit set, in comparison to up to 100 fruits set per capitulum for a compatible cross (Abbott and Forbes 1993; Hiscock, 2000a). Fruit set was recorded by counting the number of filled fruits produced per capitulum. Filled fruits, containing a fertilized ovule, appeared fatter and more pigmented than fruits containing an unfertilized ovule, which were thin and pale in colour. The strength of SI in each plant was tested by bagging capitula at the bud stage and leaving until the capitula had fully dehisced. Capitula were not emasculated prior to cross-pollination because these procedures caused excessive stigma damage. Instead, SI was relied upon to prevent the majority of illegitimate self fruit-set and an average of up to two fruits set per capitulum were scored as incompatible to allow for a low level of illegitimate self fruit-set. Indiscriminate cross-pollination was prevented by bagging capitula with pollination bags at the bud stage and rebagging them with individual perforated paper bags after pollination (Hiscock, 2000a). Cross-pollinations were carried out by gently brushing flowering capitula together. Each cross was repeated reciprocally two to six times until a full cross diallel had been achieved. Average fruit-set per capitulum was calculated and compatibility phenotypes scored according to the scheme described in the key to Figure 1.

\section{Construction and analysis of mating table diallel}

A mating table diallel was constructed based upon the compatibility phenotypes of all 26 individuals and used to identify $S$ alleles based on groupings of mutually incompatible individuals, similar to the methods described in Hiscock (2000a). It was reasoned that plants in such groups shared at least one dominantly expressed $S$ allele resulting in the same $S$ phenotype. A plant was chosen at random and all plants with which it shared an incompatible interaction were retained in its potential $S$ allele-sharing pool. From this reduced set of plants, another was chosen at random, and the grouping process repeated until an incompatibility group was formed for which every member individual had been tested in this way. A plant not belonging to this incompatibility group was then chosen at random and further incompatibility groups formed until all plants had been assigned an $S$ phenotype. $S$ phenotypes represented by only one sample plant (ie Ox19) were considered valid if that plant had already been rejected from every other incompatibility group during the grouping process.

\section{Estimating the number of $S$ alleles in a population}

Population $S$ allele number was estimated according to the equations (equations 1 and 2) used previously to estimate $S$ allele number in populations of GSI species. Because these estimators require $S$ allele data to be in a diploid genotype format, equivalent to that which would be found under GSI conditions of strict $S$ allele codominance, minimum and maximum numbers of $S$ alleles in the sample were estimated by assuming that all unidentified $S$ alleles were equivalent or that all unidentified $S$ 


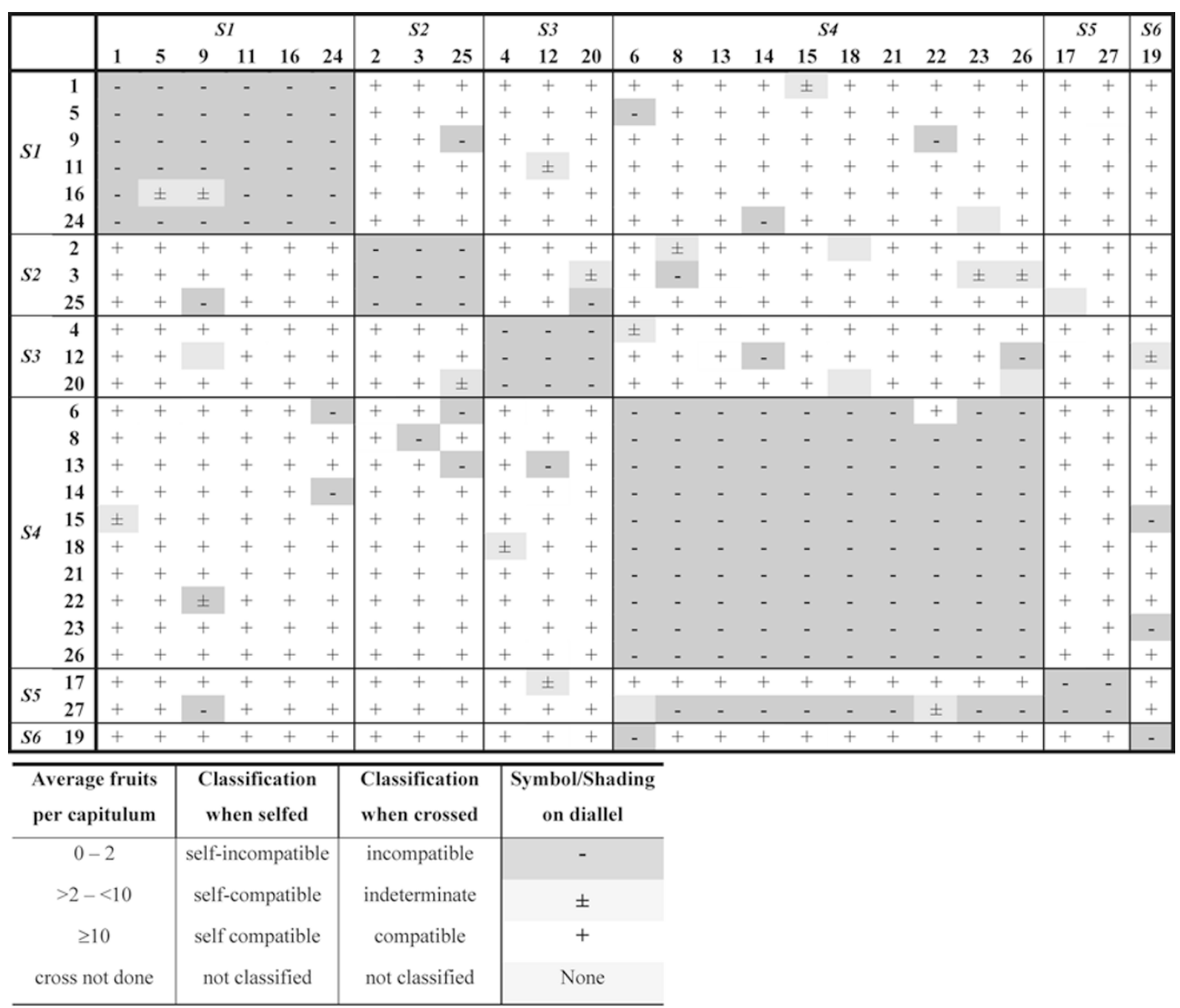

Figure 1 Analysed diallel of cross results between pairs of Senecio squalidus plants from an Oxford population. The first row and column refer to the dominantly-expressed $S$ alleles that have been identified through analysis of this mating table (see Methods). The second row and column refer to named sample individuals with plants acting as pollen donor listed across the columns and plants acting as maternal receptor listed down the rows. The rest of the diallel consists of cross results for pairs of plants, classified and colour-coded according to the average fruit-set results described in the key.

alleles were unique. This provided a range of possible $S$ allele number estimates for the population.

$$
\begin{aligned}
& \frac{n}{m}=\frac{n}{N} \log \left(\frac{1}{1-\frac{n}{N}}\right) \\
& n=N\left(1-\left(1-\frac{2}{N}\right)^{r}\right)
\end{aligned}
$$

(Whitehouse, 1949)

(Paxman, 1963)

where $n$ is the number of $S$ alleles identified in a sample, $N$ is the estimated number of $S$ alleles present in the entire population, $r$ is the number of plants sampled, and $m$ is the number of $S$ alleles sampled $(r=2 m)$.

To avoid generating the artificial minimum and maximum $S$ allele sample estimates required for the GSI estimators described above, we modified estimator equations 1 and 2 so that they could be used for SSI studies where dominance interactions between $S$ alleles generally result in the identification of a single $S$ allele per individual. These modified estimators are presented below as equations 3 and 4 (corresponding to modified versions of equations 1 and 2, respectively). Equation 4 was originally derived from equation 2 by Lawrence (1996) to estimate population $S$ allele number for the two locus GSI system found in the Poaceae, but may also be applied to the problem of estimating population $S$ allele number for SSI systems. Equation 3 was derived from equation 1 using similar principles to those used by Lawrence (1996) to modify the Paxman (1963) estimator, namely that $m$, the number of $S$ alleles sampled becomes, $r$, the number of plants sampled $(m=r)$. Similarly, the error range for the modified Paxman (1963) estimator (equation 4) can be derived from the error range for the original estimator (equation 2) presented in Paxman (1963). The assumption of equal $S$ genotype and $S$ allele frequencies at mating system equilibrium is effectively replaced with the assumption of isoplethy at mating system equilibrium. The performance of the modified Paxman (1963) estimator relative to its unmodified counterpart was tested using Monte Carlo simulations of published diploid $S$ genotype datasets and associated population $S$ allele number estimates for natural SSI populations of other species (Stevens and Kay, 1989; Kowyama et al, 1994), where the modified estimator was calculated from 1000 
resampled haploid datasets consisting of single randomly chosen $S$ alleles.

$$
\begin{aligned}
& \frac{n}{r}=\frac{n}{N} \log \left(\frac{1}{1-\frac{n}{N}}\right) \\
& n=N\left(1-\left(1-\frac{1}{N}\right)^{r}\right)
\end{aligned}
$$

(Lawrence, 1996)

Measuring the thoroughness of the SI study

Repeatability (R) is a standard measure of the thoroughness with which an $S$ allele assay has been carried out and was calculated according to equation 5 . The resulting value is a proportion ranging from zero (as many different $S$ alleles identified as $S$ alleles sampled) to one (the minimum number of $S$ alleles possible for a SSI system (ie, 2) identified in the entire sample).

$$
R=1-\frac{n-2}{r-2}
$$

(Stevens and Kay, 1989)

\section{Testing the equality of $S$ phenotype frequencies} (isoplethy)

Since the dominantly expressed $S$ alleles identified in our sample also correspond to the $S$ phenotypes, a test of the prediction of isoplethy at mating system equilibrium was appropriate. Isoplethy was tested using the $\chi^{2}$ test described in equation 6 , which is itself a modified version of the $\chi^{2}$ test developed for use in GSI studies (Campbell and Lawrence, 1981) so that it deals with single $S$ allele genotype data.

$$
\chi_{n-1}^{2}=\frac{n}{r}\left(\sum_{i=1}^{n} C_{i}^{2}-\frac{r^{2}}{n}\right)
$$

(Fearon et al, 1994)

where $C_{i}$ is the frequency of occurrence of the ith $S$ allele.

The significance of this $\chi^{2}$ statistic was evaluated according to the Monte Carlo simulation methods described by Richman et al (1996b), using $r=25$ and $n=6$.

\section{Measuring average mate availability for the sample}

An average mate availability (MA) value for the sample was calculated according to equation 7 . The resulting value is a proportion ranging from zero (all individuals inter-incompatible) to one (all individuals intercompatible).

$$
M A=\frac{1}{r} \sum_{i=1}^{r} \frac{r-C_{i}}{r-1}
$$

(Byers and Meagher, 1992)

\section{Results}

The strength of SSI in $S$. squalidus

Fruit-set counts from four to 20 selfed capitula per plant, confirmed that 25 out 26 plants in the Oxford population of $S$. squalidus were strongly SI (Table 1). One plant (Ox10) was classed as pseudo-self-compatible (PSC), according to Levin (1996), because its average fruit-set after selfing (13 fruits per capitulum, Table 1) was significantly higher than the self-fruit-set for all other plants tested but lower than the average fruit-set after crossing. All fruit-set counts based on cross pollinations involving this plant demonstrated fairly high levels of fruit-set (data not presented) so that incompatible crosses could not be identified with any certainty, making it impossible to ascribe an $S$ phenotype to this plant.

\section{Identification of $S$ alleles}

Analysis of fruit-set counts from approximately 1800 cross-pollinated capitula in a complete diallel allowed the identification of six incompatibility groups or $S$ phenotypes corresponding to six different, dominantly expressed $S$ alleles in the Oxford population (Figure 1 and Table 2). This SSI model of complete dominance satisfactorily accounts for the majority of the crossing results $(87.7 \%$, Table 3). Assuming co-dominance of alleles S4 and S5 in the stigma of plant Ox27, explains a further $2.7 \%$ of the crossing results (Table 3 ). Thus, assuming a SSI model and the dominance scheme proposed, it is possible to identify and classify 26 out of a potential 50 $S$ alleles within this sample of 25 SI plants.

\section{$S$ allele diversity}

When $S$ allele number estimators developed for GSI species (equations 1 and 2) were applied to our data for $S$. squalidus, a wide range of estimates of $S$ allele number was obtained ranging from seven to more than $34 S$ alleles for the entire Oxford population. Modified estimators were therefore developed that could take into account the dominance relationships between $S$ alleles that are characteristic of SSI (equations 3 and 4). These modified estimators predicted approximately six $S$ alleles for the entire Oxford population (without the large range associated with the GSI estimators), which was the same as the number of $S$ alleles identified in the sample. $R$ (repeatability value) was calculated as a measure of the thoroughness of $S$ allele sampling and found to be high (0.83, Table 4), indicating that much of the total $S$ allele diversity within the Oxford population was likely to be represented within our sample of 26 individuals. Different $S$ phenotypes were present at significantly unequal frequencies in the Oxford population when compared to the null hypothesis of isoplethy or equal $S$ phenotype frequencies expected at mating system equilibrium ( $\chi^{2}$ test $P$ value of 0.02 , Table 4$)$. Average mate availability, for sample individuals was high (0.78, Table 4), but likely to be depressed relative to that expected for the number of identified $S$ alleles due to unequal $S$ phenotype frequencies. A test of the performance of the modified Paxman (1963) estimator relative to its unmodified counterpart revealed that the modified estimator provided population $S$ allele number estimates very similar to the estimates provided by unmodified estimators where complete diploid $S$ genotype datasets were available (Table 5).

\section{Discussion}

\section{Low $S$ allele diversity is a feature of the SSI mating} system of $S$. squalidus in Britain

Our estimate of approximately six $S$ alleles in the Oxford population of $S$. squalidus is probably close to the true population $S$ allele number for this population because the modified estimators we used (equations 3 and 4) 
Table 1 Average self-fruit-set values, for sample individuals from an Oxford population of Senecio squalidus

\begin{tabular}{|c|c|c|c|c|c|c|c|c|c|c|c|c|c|c|}
\hline Plant & $0 \times 1$ & $0 \times 2$ & $0 \times 3$ & $O x 4$ & $0 \times 5$ & $0 \times 6$ & $0 x 8$ & $0 \times 9$ & $0 \times 10$ & $0 \times 11$ & $0 \times 12$ & Ox13 & Ox14 & Ox15 \\
\hline $\begin{array}{l}\text { Self-fruit-set value } \\
\text { (SE) }\end{array}$ & 0 & $\begin{array}{c}0.1 \\
(0.1)\end{array}$ & 0 & 0 & 0 & 0 & 0 & 0 & $\begin{array}{c}13 \\
(1.6)\end{array}$ & $\begin{array}{c}0.4 \\
(0.4)\end{array}$ & $\begin{array}{c}0.1 \\
(0.1)\end{array}$ & 0 & 0 & 0 \\
\hline Plant & Ox16 & Ox17 & Ox18 & Ox19 & $O \times 20$ & $O \times 21$ & $0 \times 22$ & $O x 23$ & $O \times 24$ & $O \times 25$ & $O \times 26$ & $O \times 27$ & All plants & All SI plants \\
\hline $\begin{array}{l}\text { Self-fruit-set value } \\
\text { (SE) }\end{array}$ & 0 & $\begin{array}{c}0.1 \\
(0.1)\end{array}$ & 0 & $\begin{array}{l}0.6 \\
(0.4)\end{array}$ & $\begin{array}{c}0.5 \\
(0.3)\end{array}$ & 0 & 0 & 0 & 0 & 0 & 0 & 0 & $\begin{array}{l}1.4 \\
(0.3)\end{array}$ & $\begin{array}{c}0.1 \\
(<0.1)\end{array}$ \\
\hline
\end{tabular}

Ox10 was classified as pseudo-self-compatible due to its high self-fruit-set. Two average self-fruit-set values were calculated, one for all individuals to give an average self-fruit-set value for the Oxford population, and one for self-incompatible individuals only, excluding Ox10, to give a strictly SI self-fruit-set value.

Table $2 S$ allele designation for Senecio squalidus individuals in the Oxford sample, based on a sporophytic self-incompatible $S$ allele dominance interpretation of the analysed diallel presented in Figure 1

\begin{tabular}{|c|c|c|c|c|c|c|}
\hline$S$ allele designation & S1 & S2 & S3 & $S 4$ & S5 & $S 6$ \\
\hline Plant & $\begin{array}{l}\text { Ox1 } \\
\text { Ox5 } \\
\text { Ox9 } \\
\text { Ox11 } \\
\text { Ox16 } \\
\text { Ox24 }\end{array}$ & $\begin{array}{l}\text { Ox2 } \\
\text { Ox3 } \\
\text { Ox25 }\end{array}$ & $\begin{array}{l}\text { Ox4 } \\
\text { Ox12 } \\
\text { Ox20 }\end{array}$ & $\begin{array}{l}\text { Ox6 } \\
\text { Ox8 } \\
\text { Ox13 } \\
\text { Ox14 } \\
\text { Ox15 } \\
\text { Ox18 } \\
\text { Ox21 } \\
\text { Ox22 } \\
\text { Ox23 } \\
\text { Ox26 } \\
\text { Ox27( }\end{array}$ & $\begin{array}{l}\text { Ox17 } \\
\text { Ox27 }\end{array}$ & Ox19 \\
\hline
\end{tabular}

Two $S$ alleles could be assigned to plant $\mathrm{Ox} 27$ as its $S$ alleles interacted in a partially codominant manner. $P$ associated with the $S 4$ allele of plant Ox27 refers to this $S$ allele's codominance interaction with allele $S 5$ in maternal stigma tissue but recessiveness to $S 5$ in pollen.

Table 3 Summary statistics for cross-classifications and the explanatory power of the sporophytic self-incompatibility model of near-complete $S$ allele dominance interactions for the analysed diallel of cross results in the Oxford Senecio squalidus population

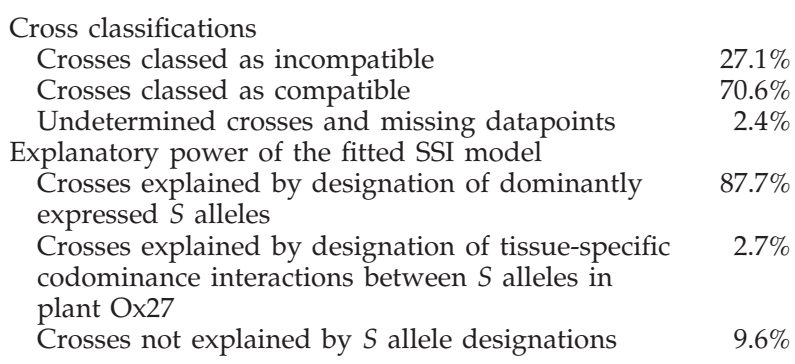

allowed for dominant $S$ allele interactions of the type encountered in a species with SSI. The modified estimators permit the estimation of population-level $S$ allele diversity without recourse to the minimum and maximum estimates of $S$ allele number required by estimators used for GSI species (equations 1 and 2). GSI estimators unavoidably and greatly inflate the error bounds of the resulting estimates of population $S$ allele number,
Table 4 Analysis of $S$ allele diversity based upon $S$ allele designations derived from a sporophytic-self-incompatible interpretation of classified cross results for an Oxford Senecio squalidus population. Equations 1-7 are described in the methods

\begin{tabular}{|c|c|c|}
\hline Parameter & Estimator & $\begin{array}{c}\text { Value } \\
\text { (range of values) }\end{array}$ \\
\hline \multirow{4}{*}{$\begin{array}{l}\text { Number of } S \text { alleles estimated to } \\
\text { be in entire population }(n)\end{array}$} & Eq. 1 & $7.01-34.08$ \\
\hline & Eq. 2 & $7.00-33.19$ \\
\hline & Eq. 3 & 6.10 \\
\hline & Eq. 4 & $6.05-6.09$ \\
\hline Thoroughness of study (R) & Eq. 5 & 0.83 \\
\hline$\chi^{2}$ test of isoplethy & Eq. 6 & $13.16(P=0.02)$ \\
\hline Average mate availability (MA) & Eq. 7 & 0.78 \\
\hline
\end{tabular}

but the modified estimators rely on less stringent assumptions about $S$ allele frequencies and distributions in populations. Also, the modified estimators merely assume equal $S$ phenotype frequencies, including the possibility of $S$ homozygotes, rather than assuming the equal $S$ genotype frequencies and strict $S$ heterozygosity required by the unmodified GSI estimators. Monte Carlo simulations of resampled diploid $S$ genotype datasets for other SSI species (Sinapis arvensis and Ipomoea trifida) show that the modified Paxman (1963) estimator (equation 4) performs very well in comparison to its unmodified counterpart (equation 2) since the two estimators provide equivalent population $S$ allele estimates, despite resampled haploid datasets being less informative than the original diploid datasets (Table 5). This is good corroborating evidence that the modified estimator has been appropriately applied and that the number of $S$ alleles in the Oxford population of $S$. squalidus is close to the estimate of six $S$ alleles provided by the modified estimators.

A population $S$ allele estimate, equivalent to the sample estimate provided by the modified estimators but lower than the minimum estimate provided by the unmodified GSI estimator, is a consequence of no longer needing to assume the presence of a universal unidentified recessive $S$ allele (ie, S7). As part of a normally functioning SSI system, all $S$ alleles should have representative $S$ phenotypes at isoplethy within populations such that the most recessive $S$ allele in a dominance series will not only be present at high frequency but will also be expressed as an $S$ phenotype class of its own, composed 
Table 5 Results of Monte Carlo simulations to compare the performance of modified population $S$ allele estimators relative to unmodified counterparts using 1000 haploid $S$ genotype datasets resampled from previously published diploid $S$ genotype datasets for natural populations of two SSI species Sinapis arvensis and Ipomoea trifida. Equations 2 and 4 are described in the methods

\begin{tabular}{|c|c|c|c|c|}
\hline SSI species & $\begin{array}{l}\text { Sample size } \\
(m)\end{array}$ & $\begin{array}{c}\text { Number of identified } S \\
\text { alleles }(n)\end{array}$ & $\begin{array}{c}\text { Population } S \text { allele number } \\
\text { estimate }(N ; E q .2)\end{array}$ & $\begin{array}{l}\text { Population } S \text { allele number estimate } \\
\left(N_{\text {mod. }}: E q .4\right)(S . E .)\end{array}$ \\
\hline Sinapis arvensis & $35^{\mathrm{a}}$ & $35^{\mathrm{a}}$ & 43.24 & $40.56(1.88)$ \\
\hline Ipomoea trifida & $40^{\mathrm{b}}$ & $21^{b}$ & 21.42 & $18.47(0.49)$ \\
\hline
\end{tabular}

andividual $S$ genotype scorings for a South Wales population (Stevens and Kay, 1989).

bIndividual $S$ genotype scorings for population G81 (Kowyama et al, 1994).

entirely of $S$ homozygous individuals (Byers and Meagher, 1992, Schierup et al 1997).

The finding of non-isoplethy of $S$ phenotypes ( $\chi^{2}$ test, $P=0.02$, Table 4$)$ is worth considering in the context of the assumptions of the modified estimators. In general, non-isoplethy results in underestimation of population $S$ allele number, since some $S$ alleles will be rare and less likely to be represented in samples (Campbell and Lawrence, 1981, Lawrence, 2000). Unequal $S$ allele and $S$ phenotype frequencies have previously been observed in natural populations of SSI Sinapis arvensis (Stevens and Kay, 1989) and GSI Papaver rhoeas (Campbell and Lawrence, 1981). Some of the possible reasons for non-isoplethy, which were thoroughly investigated in P. rhoeas, include: populations not being at mating system equilibrium; stochastic variation in frequencies at mating system equilibrium; and selection at loci linked to the $S$ locus (Brooks et al, 1996; Lawrence, 2000). Non-isoplethy of $S$ phenotypes in $S$. squalidus could also be attributable to either non-equilibrium mating system in highly anthropogenic S. squalidus habitats or stochastic variation in sampling frequencies due to such factors such as unequal plant size. However, since the absence of mating system equilibrium cannot be distinguished from the range of other causes of non-isoplethy without extensive further study of the samples, a population $S$ allele estimator dependent on isoplethy is considered reasonable until more detailed knowledge of long term mating dynamics in $S$. squalidus becomes available.

The preference for an estimate of close to six $S$ alleles in the Oxford S. squalidus population, based on the greater theoretical applicability of modified estimators rather than the original GSI estimators, is supported by other independent sources of evidence. The high proportion of incompatible crosses $(27.1 \%$, Table 3$)$ in the cross diallel, provide an intuitive indication that many individuals in the sample share $S$ alleles (Bateman, 1947). A similar proportion of incompatible crosses $(34.5 \%)$ were found in a previous study of SI in British S. squalidus (Abbott and Forbes, 1993). The high repeatability value for the present study ( $R=0.83$, Table 4$)$, corresponding to few $S$ alleles identified relative to the number of $S$ alleles sampled, suggests that 26 plants was probably enough to represent all the $S$ allele diversity in the Oxford population. In a previous study of SSI in S. squalidus, Hiscock (2000a) identified as few as three $S$ alleles, with one universal recessive $S$ allele, in four $S$. squalidus individuals sampled from locations several kilometres apart in Oxford, based on crosses between force-selfed progeny arrays (Hiscock $2000 \mathrm{a}, \mathrm{b})$, which is further evidence that very few $S$ alleles are present in the British population.

An estimate of six $S$ alleles in $S$. squalidus is consider- ably lower than previous estimates made for other SSI species, such as Brassica campestris, 22 and $31 S$ alleles for two different populations (Nou et al, 1993), and Sinapis arvensis, $43 S$ alleles for a single population (Stevens and Kay, 1989). Low $S$ allele number in $S$. squalidus is probably a consequence of the extreme population bottleneck conditions predicted for the species during its introduction and establishment in the British Isles (Abbott and Forbes, 1993; Hiscock, 2000b). Unfortunately, important details about the introduction of $S$. squalidus such as the size of the initial founder population of $S$. squalidus introduced into the Oxford Botanic Garden and how plants were maintained and propagated before the 'escape' and subsequent dramatic population expansion during its spread away from Oxford are unknown (Harris, 2002). Nevertheless, detailed knowledge of the dynamics of the subsequent colonization of $S$. squalidus throughout Britain is available and further elucidation of this process is ongoing (Harris, personal communication).

\section{Mating system consequences of low $S$ allele diversity}

Since $S$. squalidus appears to have fewer $S$ alleles than are generally found at evolutionary mating system equilibrium for SSI, mate availability (MA) may restrict seed-set (Byers and Meagher, 1992). The fact that non-isoplethy of $S$ phenotypes is a feature of the Oxford population does not appear to be reducing MA much further than low $S$ allele number alone, since if the MA value calculated for the Oxford sample $(77.6 \%$, Table 4$)$ is representative of the entire Oxford population of S. squalidus, then it is close to the deterministic MA value $(83.3 \%)$ predicted for SSI populations consisting of six $S$ alleles interacting to form a simple dominance series (Schierup et al, 1997). Furthermore, ecological factors such as small, variable population size, population substructure, or unequal reproductive capabilities, all of which are features of natural S. squalidus populations and which might serve to depress MA further, do not appear to be having a significant effect on reproductive assurance. The success of S. squalidus as a colonizing species in Britain suggests that low MA does not restrict the reproductive potential of $S$. squalidus. Other characteristics of the mating behaviour of $S$. squalidus may be sufficient to explain why sexual reproduction is not adversely affected by low MA in natural populations. The capitulum of $S$. squalidus consists of approximately 80-100 individual flowers with centripetal maturation over the course of about 1-2 weeks. This long flowering period for individual capitula under natural conditions makes multiple pollinator visits and fertilization opportunities a regular occurrence. Furthermore, each plant produces many capitula over the course of a long flowering season between April and 
October (although, in Britain, sporadic flowering continues from November to March), so maximising the potential compatible mate pairing within a population for any given year. Mating potential is further optimized by the perennial habit (albeit short-lived) of $S$. squalidus. Nevertheless, despite these potential compensatory life history traits for low $S$ allele diversity, the SSI system of S. squalidus itself is likely to have been subject to selective pressures to improve reproductive assurance since its introduction to Britain. Possible adaptive solutions to the problem of low $S$ allele diversity include: (i) breakdown of SI leading to the evolution of self-compatibility (SC); (ii) evolution of new $S$ alleles; (iii) introgression of $S$ alleles into $S$. squalidus from other Senecio species; (iv) alteration of dominance interactions between $S$ alleles; and (v) evolution at loci that modify SSI expression. We will now consider the potential contribution made by each of these evolutionary scenarios to the maintenance of SSI in S. squalidus.

(i) Breakdown of SI leading to the evolution of SC: Theory suggests that SC will evolve from SI whenever there is variation in the strength of expression of SI and the selective advantage of avoiding inbreeding depression under $\mathrm{SI}$ is less than twice the selective advantage of reproductive assurance under SC (Uyenoyama, 1986; Levin, 1996). Mutations that disrupt $S$ allele activity, either directly in the form of self-sterility alleles or indirectly in the form of modifier loci causing pseudo-self-compatibility (PSC), are sufficient to provide variation in SI expression for selection to act upon. Population events that perturb the mating system and alter the selective balance between SI and SC, such as the purging of genetic load during population bottlenecks or the increased selective advantage of reproductive assurance during colonization may readily initiate a breakdown of SI (Levin, 1996). Within the Asteraceae, recent breakdown of SI has been documented in Aster furcatus as a result insufficient $S$ allele diversity resulting from declining population size (Reinartz and Les, 1994). Even so, exceptions to this course of events are common and SI systems often survive intact through dramatic population events such as the introduction and spread of Centaurea solstitialis in western USA (Sun and Ritland, 1998) or the decline and fragmentation of Hymenoxys acaulis var. glabra populations (DeMauro, 1993). There is no evidence that SSI is breaking down in S. squalidus since British populations clearly maintain a strong, fully functional SSI system (Abbott and Forbes, 1994; Hiscock, 2000a, b, Brennan and Hiscock, unpublished). A simple model of SSI involving dominance and limited tissue-specific codominance between $S$ alleles satisfactorily explains the majority of cross results $(90.4 \%$, Table 3) in our sample diallel, demonstrating that SSI in S. squalidus functions as expected. Most of the plants sampled from the wild were unambiguously SI when tested in the greenhouse (Table 1). Nevertheless, one PSC plant (Ox10), (average selffruit-set $=13$, Table 1 ) was identified in the Oxford sample and a low frequency of PSC plants can be found in other British populations of S. squalidus (1.33\%, Brennan and Hiscock, unpublished). This may be evidence of selection acting to increase compatible matings, although the fact that the frequency of PSC is neither high nor approaching fixation implies that selection continues to favour the maintenance of SI over PSC or SC. (ii) Evolution of new $S$ alleles: The possibility that new $S$ alleles may have evolved in British S. squalidus is unlikely given the relatively short period of time that has elapsed since its introduction to Britain, c. 300 years ago (approximately 300 generations). New $S$ alleles are thought to evolve only under a limited range of evolutionary scenarios, such as the temporary evolution of dual specificity $S$ alleles (Matton et al, 1999), or temporary breakdown of SI (Uyenoyama et al, 2001). Ancient $S$ polymorphisms, often predating speciation, are typical of SI systems and further emphasise the rarity with which new $S$ alleles arise (Ioerger et al, 1990; Richman et al, 1996a). The rate of evolution of new $S$ alleles is responsive to increased selection pressures as indicated by accelerated rates of $S$ allele diversification in Physalis crassifolia in response to population bottleneck events, but even this 'rapid' recovery of the SI system was on the scale of tens of thousands of generations post-population recovery (Richman et al, 1996b). Senecio squalidus provides a rare opportunity to test hypotheses on the short-term limits of $S$ allele evolution by searching for unique 'British' $S$ alleles in comparative surveys of $S$ allele diversity in British and Sicilian populations.

(iii) Introgression of $\mathrm{S}$ alleles into $\mathrm{S}$. squalidus from other Senecio species: It is possible that the success of $S$. squal$i d u s$ as a colonizer in Britain may have been the result of introgression of new $S$ alleles from other Senecio species during cultivation in the years since the introduction of S. squalidus to Britain, which could then account for the rapid spread of S. squalidus following a period of 150 years spent confined to the city walls of Oxford. This extra $S$ allele diversity may then have been sufficient to alleviate any SI-related reproductive constraints and allow rapid range expansion beyond Oxford. There are no genetic barriers to hybridisation between $S$. squalidus and its putative parental taxa $S$. chrysanthemifolius and $S$. aethnensis and gene flow from these species into $S$. squalidus has been demonstrated by isozyme analyses of the three species on Mt. Etna, Sicily (Abbott et al, 2000). So much greater the potential then, for introgression of extra $S$ alleles into $S$. squalidus if these and related Senecio species were cultivated together in the Duchess of Beaufort's gardens at Badminton or the Oxford Botanic Gardens (Harris, 2002). Hybridisation events between S. squalidus and native Senecio species, such as S. vulgaris, have also been recorded (Abbott, 1992). However, since S. vulgaris is self-compatible it is unlikely to have contributed extra $S$ alleles directly to $S$. squalidus except possibly in the form of pseudogenes retained in the genome from an ancestral SI form. Certainly any newly introgressed $S$ alleles would be subject to strong negative frequency dependent selection and likely to be maintained in populations even if the hybridizations were rare or resulted in hybrids of initially low fertility.

(iv) Alteration of dominance interactions between $S$ alleles: The $S$ alleles identified in the Oxford population sample demonstrated a high degree of dominance, with all but one of the sample plants (Ox27) expressing completely dominant $S$ alleles with no evidence for the presence of fully codominant $S$ alleles (Figure 1 and Table 2). This may be an adaptive feature of the SSI system in British populations of $S$. squalidus in response to low $S$ allele diversity. The possibility of selectively altering domi- 
nance interactions between $S$ alleles in SSI systems provides an extra dimension of 'flexibility' to the SI system during times of population perturbation of the kind experienced by $S$. squalidus. In general, increased dominance between $S$ alleles will result in increased MA relative to that predicted under a codominant SSI system (Byers and Meagher, 1992, Schierup et al, 1997).

This is a consequence of individual mating phenotypes being reduced to that of their dominant $S$ allele alone, thereby increasing the MA by the proportion of plants in the population expressing the recessive $S$ allele. Compatible crosses between close relatives, such as full sibs or parents and their progeny, become a regular feature of SSI in the presence of high levels of dominance between $S$ alleles and have a pronounced effect on mating system dynamics. In particular, populations will experience a degree of inbreeding and its associated evolutionary implications (Uyenoyama, 1986), even in the presence of a fully functional SSI system. Allozyme studies have provided evidence for inbreeding in S. squalidus. Abbott et al (2000) calculated an $F_{\text {IS }}$ (inbreeding coefficient) value as high as 0.22 but our allozyme data for the Oxford Population shows a lower but significant $F_{\text {IS }}$ value of 0.03 (significantly greater than zero; $P<0.01$; Brennan, unpublished data). Since, SI effectively limits selfing in the Oxford population (Table 1), inbreeding must be predominantly due to matings between close relatives where $S$ allele dominance interactions allow.

Another potential benefit arising from increased compatibility between close relatives as a consequence of high levels of dominance between $S$ alleles, is the potential improvement of a SSI species' colonizing ability during normal metapopulation dynamics. Single SI individuals still cannot found new populations (Pannell and Barrett, 1998), but as few as two $S$ alleles in two or more compatible individuals will be sufficient to found a selfsustaining population (Schierup et al, 1997). Thus, increased dominance interactions between $S$ alleles is a potential feature of SSI species that have experienced selection in response to colonisation. Further research to elucidate the particular dominance relationships between different $S$ alleles in $S$. squalidus and comparison of these $S$ allele interactions between British, Sicilian and other European populations may provide valuable insights into the evolution of $S$ allele dominance interactions in SSI systems.

(v) Evolution at loci that modify SSI expression: Another evolutionary response of the SI system to selective pressures imposed by loss of $S$ allele diversity could be evolutionary change at genetic loci that influence expression of SI - so called modifier loci, unlinked to the $S$ locus (Levin, 1996). In general, these loci specifically suppress $S$ allele expression resulting in SC, PSC or $S$ phenotypes differing from those predicted by the genetics of the $S$ locus alone and are hypothesised to provide SI systems with more flexible patterns of expression than are otherwise possible at a single genetic locus (Levin, 1996). PSC was observed in the offspring of some individuals of $S$. squalidus following forced selfing (Hiscock, 2000a, b) and its presence in natural populations has been explained as a consequence of selection for reproductive assurance during population bottlenecks or colonisation events (Levin, 1996). In SSI Brassica there is evidence that a cryptic system of GSI is operational and influences compati- bility in certain genetic backgrounds and combinations of $S$ alleles (see Lewis, 1994 for review). This cryptic GSI system is regulated by a single locus, $G$, with just two alleles, and generally leads to the occurrence of 'anomalous' compatibilities where the $S$ allele phenotype would predict incompatibility. It is possible that a similar cryptic $G$ gene system is also operational in S. squalidus and is responsible for the anomalous compatibilities observed in genetic analyses of SSI (Hiscock, 2000a, b). The activity of this locus might offer an explanation for some of the 9.6\% 'anomalous' crosses identified in the diallel that remain unexplained by a sporophytic model of SI (Table 3).

Data presented here indicate that an Oxford population of $S$. squalidus contains approximately six $S$ alleles. This population-level estimate of $S$ allele number sets a new lower limit on the range of population $S$ allele number estimates for species with SSI which previously ranged from 22 to $43 S$ alleles. It is argued that the low $S$ allele estimates for $S$. squalidus may be a consequence of its unusual population history in the British Isles. The finding of non-isoplethy for $S$ phenotype frequencies suggests that the Oxford population is not at mating system equilibrium and implies that high levels of disturbance continue to characterise British populations of $S$. squalidus. Low $S$ allele diversity is likely to have significant consequences for mating dynamics, but SSI has clearly not broken down in British $S$. squalidus nor is it likely that extra, compensatory $S$ allele diversity, in terms of $S$ allele number, has evolved yet in British populations of $S$. squalidus. Other features of the SSI system of S. squalidus would seem more likely to account for the maintenance of a fully effective SI system with relatively few $S$ alleles. These features include a high level of dominance observed between $S$ alleles, the influence of modifier loci, and the reproductive life history of $S$. squalidus.

\section{Acknowledgements}

The work described in this paper was supported primarily by a BBSRC studentship to ACB and a BBSRC David Phillips Research Fellowship to SJH. ACB also gratefully acknowledges additional funding from: Department of Plant Sciences, University of Oxford; a Mark Quested Award, Fishmongers' Company, London; a Margaret Pollock Award, Somerville College, Oxford; and The Lawlor Foundation, Belfast. We also wish to thank Christine Surman and Phil Smith for unceasing plant care, Michael J Lawrence and Yan Wong for valuable discussion, and two anonymous reviewers for constructive comments on earlier drafts of this paper.

\section{References}

Abbott RJ (1992). Plant invasions, interspecific hybridisation and the evolution of new plant taxa. Trends Ecol Evol 7: 401-405.

Abbott RJ, Forbes DG (1993). Outcrossing rate and self-incompatibility in the colonising species Senecio squalidus. Heredity 71: 155-159.

Abbott RJ, James JK, Irwin JA, Comes HP (2000). Hybrid origin of the Oxford Ragwort, Senecio squalidus L. Watsonia 23: 123-138.

Bateman AJ (1947). Number of $S$ alleles in a population. Nature 160: 337.

Brooks RJ, Tobias AM, Lawrence MJ (1996). The population genetics of the self-incompatibility polymorphism in Papaver 
rhoeas XI. The effects of limited pollen and seed dispersal, overlapping generations and variation in plant size on the variance of $S$ allele frequencies in populations at equilibrium. Heredity 76: 367-376.

Byers DL, Meagher TR (1992). Mate availability in small populations of plant species with homomorphic self-incompatibility. Heredity 68: 353-359.

Campbell JM, Lawrence MJ (1981). The population genetics of the self-incompatibility polymorphism in Papaver rhoeas II. The number and frequencies of $S$ alleles in a natural population R106. Heredity 46: 81-90.

Demauro MM (1993). Relationship of breeding system to rarity in the lakeside daisy (Hymenoxys acaulis var. glabra). Conserv Biol 7: 542-550.

Fearon CH, Cornish MA, Haywood MD, Lawrence MJ (1994). Self-incompatibility in ryegrass $\mathbf{X}$. Number and frequency of alleles in a natural population of Lolium perenne L. Heredity 73: 254-261.

Finney DJ (1952). The equilibrium of a self-incompatible polymorphic species. Genetica 26: 33-64.

Harris SA (2002). Introduction of Oxford Ragwort, Senecio squalidus L. (Asteraceae), to the United Kingdom. Watsonia 24: $31-43$

Hiscock SJ (2000a). Genetic control of self-incompatibility in Senecio squalidus L. (Asteraceae): a successful colonizing species. Heredity 85: 10-19.

Hiscock SJ (2000b). Self-incompatibility in Senecio squalidus L. (Asteraceae). Ann Bot 85 (Suppl A): 181-190.

Hiscock SJ (2002). Pollen recognition during the self-incompatibility response in plants - the ties that bind. Genome Biol 3: 1004.1-1004.6.

Hiscock SJ, Kues U (1999). Cellular and molecular mechanisms of sexual incompatibility in plants and fungi. Int Rev Cytol 193: 165-295.

Ioerger TR, Clark AG, Kao T-H (1990). Polymorphism at the selfincompatibility locus in the Solanaceae predates speciation. Proc Natl Acad Sci 87: 9732-9735.

Kowyama Y, Takahasi H, Muraoka K, Tani T, Hara K, Shiotani I (1994). Number, frequency and dominance relationships of $S$ alleles in diploid Ipomoea trifida. Heredity 73: 275-283.

Lawrence MJ (1996). Number of incompatibility alleles in clover and other species. Heredity 76: 610-615.

Lawrence MJ (2000). Population genetics of the homomorphic SI polymorphism in flowering plants. Ann Bot 85 (Suppl A). 221-226.

Levin DA (1996). The evolutionary significance of pseudo-selffertility. Am Nat 148: 321-332.
Lewis D (1994). Gametophytic-sporophytic incompatibility. In: Williams EG, Clarke AE, Knox RB (eds) Genetic Control of Selfincompatibility and Reproductive Development in Flowering Plants. Kluwer: Dordrecht. pp 88-101.

Matton DP, Luu DT, Xike Q, Laublin G, O'Brien M, Maes O et al (1999). Production of an S-Rnase with dual specificity suggests a novel hypothesis for the generation of new $S$ alleles. Plant Cell 11: 2087-2098.

Nou IS, Watamanbe M, Isogai A, Hinata K (1993). Comparison of $S$-alleles and $S$-glycoproteins between two wild populations of Brassica campestris in Turkey and Japan. Sex Plant Repro 6: 79-86.

Pannell JR, Barrett SCH (1998). Baker's law revisited: reproductive assurance in a metapopulation. Evolution 52: 657-668.

Paxman GJ (1963). The maximum likelihood estimation of the number of self-sterility alleles in a population. Genetics 48: 1029-1032.

Reinartz JA, Les DH (1994). Bottleneck induced dissolution of self-incompatibility and breeding system consequences in Aster furcatus (Asteraceae). Am J Bot 81: 446-455

Richman AD, Uyenoyema MK, Kohn JR (1996a). Allelic diversity and gene genealogy at the self-incompatibility locus in the Solanaceae. Science 273: 1212-1216

Richman AD, Uyenoyama MK, Kohn JR (1996b). S-allele diversity in a natural population of Physalis crassifolia (Solanaceae) (ground cherry) assessed by RT-PCR. Heredity 76: 497-505.

Schierup MH, Vekemans X, Christiansen FB (1997). Evolutionary dynamics of self-incompatibility alleles in plants. Genetics 147: 835-846.

Stevens JP, Kay QON (1989). The number, dominance relationships and frequencies of self-incompatibility alleles in a natural population of Sinapis arvensis L. in South Wales. Heredity 62: 199-205.

Sun M, Ritland K (1998). Mating system of yellow starthistle, (Centaurea solstitalis): a successful colonizer in North America. Heredity 80. 225-232.

Uyenoyama MK (1986). Inbreeding and the cost of meiosis: the evolution of selfing in populations practicing biparental inbreeding. Evolution 40: 388-404.

Uyenoyema MK, Zhang Y, Newbigin E (2001). On the origin of self-incompatibility haplotypes: transition through self compatible intermediates. Genetics 157: 1807-1817.

Whitehouse HLK (1949). Multiple allelomorph heterothallism in the fungi. New Phytol 48: 212-244.

Wright S (1939). The distribution of self-sterility alleles in populations. Genetics 24: 538-552. 